\title{
La controversia Pasteur vs. Pouchet sobre la generación espontánea: un recurso para la formación inicial del profesorado en la naturaleza de la ciencia desde un enfoque reflexivo
}

\author{
The Pasteur-Pouchet controversy about spontaneous \\ generation: a resource for initial teacher training \\ on the nature of science from a reflective approach
}

José Antonio Acevedo-Díaz • Antonio García-Carmona ${ }^{2}$ • María del Mar Aragón ${ }^{3}$

\begin{abstract}
Resumen: Se presenta la controversia entre Pasteur y Pouchet sobre la generación espontánea como un relato de la Historia de la Ciencia interesante para abordar en las clases de ciencias algunas cuestiones de Naturaleza de la Ciencia (NDC), desde un enfoque explícito y reflexivo. La propuesta se dirige a la formación de Estudiantes de Profesorado de Educación Secundaria (EPES) en NDC y su didáctica. Se presta atención tanto a los aspectos epistémicos como a los no-epistémicos en el texto de la controversia y en las cuestiones que se plantean. Asimismo, se proponen algunas recomendaciones metodológicas para su implementación y evaluación en el aula, que se concreta en: (1) lectura de la controversia y respuesta en equipo a unas cuestiones sobre NDC; (2) discusión en clase de las primeras respuestas; y (3) revisión de las respuestas iniciales a partir de la discusión en clase.
\end{abstract}

Palabras clave: Historia de la ciencia. Naturaleza de la ciencia. Formación del profesorado. Pasteur. Pouchet. Generación espontánea.

\begin{abstract}
The Pasteur-Pouchet controversy about spontaneous generation is presented as an interesting story from the History of Science to learn about the Nature of Science (NOS). The aim is to use this controversy for addressing some NOS issues in science education from an explicit and reflective approach. The didactic proposal is focused on the pre-service Secondary Education science teachers training in NOS and its teaching. Attention is given to both epistemic and non-epistemic aspects in the text of the controversy and the NOS questions asked. In addition, finally some methodological recommendations for implementing and assessing the didactic proposal in science classroom are offered. This involves the following: (1) in small groups, the students read the controversy and respond to some questions on NOS; (2) they present their responses to the whole class; and (3) they revise their initial responses in light of the whole-class discussion.
\end{abstract}

Keywords: History of science. Nature of science. Teacher training. Pasteur. Pouchet. Spontaneous generation.

\footnotetext{
${ }^{1}$ Inspector de Educación jubilado, Huelva, España. E-mail: <ja_acevedo@vodafone.es>.

${ }^{2}$ Universidad de Sevilla, Facultad de Ciencias de la Educación, Departamento de Didáctica de las Ciencias Experimentales y Sociales, Sevilla, España.

${ }^{3}$ Universidad de Cádiz, Departamento de Didáctica, Cádiz, España.
} 


\section{Introducción: naturaleza de la Ciencia e historia de la Ciencia}

Los currículos escolares de ciencia suelen centrarse en los contenidos conceptuales y se rigen por la lógica interna de la ciencia, pero se olvidan de dar formación sobre la ciencia misma; i.e., qué es la ciencia, cómo funciona y se desarrolla, cuáles son sus fundamentos epistemológicos y ontológicos, los rasgos del trabajo de los científicos, sus valores como grupo social, así como las influencias mutuas entre ciencia, tecnología y sociedad. Estos asuntos, entre otros, constituyen lo que se conoce como Naturaleza de la Ciencia (NDC).

La NDC es un metaconocimiento sobre la ciencia, que surge de las reflexiones interdisciplinares hechas por expertos en filosofía, historia y sociología de la ciencia, así como por algunos científicos y educadores de ciencias. Una definición de la NDC con precisión es difícil; aunque, en general, puede decirse que trata de aquello que caracteriza a la ciencia como una forma especial de construcción de conocimiento (ACEVEDO-DÍAZ; GARCÍA-CARMONA, 2016a). La NDC se refiere también a los valores propios y contextuales de la ciencia (ACEVEDO-DÍAZ, 1998; ECHEVERRÍA, 2002) y a los supuestos subyacentes al conocimiento científico, que son consecuencia del carácter humano de la propia actividad científica, incluyendo sus limitaciones e influencias de todo tipo.

La demanda de la Historia de la Ciencia (HDC) en la educación científica se remonta a la segunda mitad del siglo XIX. Por ejemplo, Jenkins (1990, p. 274, traducción nuestra) menciona que George J. D. Campbell, octavo duque de Argyll, en su discurso presidencial de la British Association for the Advancement of Science de la reunión de 1855, celebrada en Glasgow, reclamó que: "Lo que queremos en la enseñanza de los jóvenes no es tanto meros resultados sino métodos y, sobre todo, historia de la ciencia." Desde entonces, han sido numerosos los intentos por introducir la HDC en la educación científica apelando a motivos diversos (véase, e.g., MCCOMAS, 2013). Uno de ellos es su potencial para el aprendizaje de aspectos de NDC.

Los National Science Education Standards de EE.UU. (UNITED STATES, 1996) recomendaron, hace dos décadas, la incorporación de la HDC en la educación científica para mejorar la comprensión de la NDC. Poco después, la tesis doctoral de Abd-El-Khalick (1998) mostró que los cursos de HDC tienen un efecto pequeño en la mejora de la comprensión de aspectos de NDC, a menos que estos se impartan con un enfoque explícito y reflexivo (ABD -EL-KHALICK; LEDERMAN, 2000). Si los estudiantes no tienen la oportunidad explícita de relacionar reflexivamente un relato de HDC con aspectos de NDC, lo más probable es que no mejoren sus concepciones de NDC (ABD-EL-KHALICK, 2013; MCCOMAS, 2008).

Actualmente, se sugiere la incorporación de la HDC a la ciencia escolar en los currículos de muchos países (EUROPEAN COMISSION, 2011; NEXT GENERATION SCIENCE STANDARDS, 2013). No obstante, de los dos documentos citados, el uso de la HDC como contexto adecuado para aprender NDC solo se recomienda de manera explícita en los estándares estadounidenses. Asimismo, la integración de la HDC en las clases de ciencia es un reto aún por conseguir (CONFEDERACIÓN DE SOCIEDADES CIENTÍFICAS DE ESPAÑA, 2011; MCCOMAS; KAMPOURAKIS, 2015). 


\section{Las controversias de HDC como recurso para la enseñanza de NDC}

Un enfoque basado en la HDC permite la contextualización explícita de la enseñanza de diversos aspectos de NDC (ABD-EL-KHALICK 1999; CLOUGH, 2011); e.g., cuestiones relacionadas con la forma en que los científicos se enfrentan a sus investigaciones, o el papel que tiene la comunidad científica en la construcción de las ideas científicas y el ajuste de estas a la evidencia empírica. De este modo, se ilustran cuestiones de epistemología, ontología y sociología de la ciencia ligadas a la comprensión de la NDC (MCCOMAS, 2008), situando el contenido de la ciencia en un contexto humano, social y culturalmente más amplio. Esta perspectiva humanista de la ciencia favorece el conocimiento de la influencia de los factores contextuales sobre el pensamiento y las investigaciones de los científicos (MATTHEWS, 2015). Como sucede con las grandes obras literarias, el conocimiento científico es un producto cultural y, al igual que en ellas, la importancia de sus logros y la comprensión de su naturaleza se ven reforzados por el conocimiento de su contexto histórico (MONK; OSBORNE, 1997). En suma, la comprensión de los estudiantes sobre NDC puede mejorar por medio del uso adecuado de narraciones de HDC en el aula (MATTHEWS, 2015; MCCOMAS, 2008).

La utilización de la HDC se justifica también por el efecto beneficioso que puede tener en la participación de los estudiantes para aprender NDC, si se plantea con un enfoque explícito y reflexivo (ACEVEDO-DÍAZ, 2009). Las narraciones de HDC deben hacerse mediante descripciones detalladas para evitar una visión demasiado lineal sobre cómo la ciencia produce sus conocimientos, y para permitir una mejor interpretación contextual de la HDC. Ahora bien, su uso instrumental en el aula también requiere de una adaptación, en la que se seleccionen fragmentos de HDC y se simplifiquen los hechos expuestos. En este proceso, se debe tener especial cuidado en que las omisiones no conduzcan a una pseudohistoria (ALLCHIN, 2004) y, consecuentemente, a una imagen deformada de la ciencia (FORATO; MARTINS; PIETROCOLA, 2011).

Las controversias cientificas son episodios de HDC especialmente interesantes como recurso para la enseñanza de la NDC. Para Tolvanen et al. (2014), las confrontaciones son conflictos entre dos o más teorías, mientras que los diálogos son conflictos entre personas. Ambos tipos de conflictos están incluidos en las controversias científicas, y su discusión se usa para mostrar la evolución de la ciencia mediante los debates producidos.

Una controversia científica es una disputa pública duradera sin una resolución fácil, que implica la intervención de la comunidad científica con argumentos epistémicos (propios de la ciencia) y no-epistémicos, tales como: emociones, rasgos de personalidad, presiones institucionales, influencias políticas, rivalidades nacionales, e incluso fraude. McMullin (1987, p. 51) define una controversia científica como:

[...] una disputa [científica] pública que se mantiene persistentemente sobre un asunto considerado significativo por un número de científicos en ejercicio [...] que existe solo en el caso de que grupos sustanciales de la comunidad científica atribuya el mérito a cada parte en un desacuerdo público [...] El énfasis puesto en el papel de la comunidad [científica] en la determinación de la controversia puede servir para añadir otro aspecto respecto a la naturaleza de la controversia científica. Una controversia es un suceso histórico; 
tiene un lugar y una fecha. No se trata de una mera relación abstracta entre evidencia e hipótesis.

El mismo autor establece también cuatro tipos de controversia científica: (i) controversias en torno a hechos, que se originan como una consecuencia de los resultados obtenidos y su interpretación; (ii) controversias relativas a teorías, que surgen de desacuerdos sobre aspectos teóricos; (iii) controversias referidas a principios, que se deben a la confrontación de ciertos aspectos metodológicos y ontológicos subyacentes en toda actividad investigadora; y (iv) controversias mixtas, en las que confluyen diversos ámbitos sociales, tales como ciencia, aplicaciones tecnológicas, economía, política, moral y ética, etc.

Las controversias científicas son esenciales en la construcción del conocimiento científico, porque impulsan el avance de la ciencia y muestran el conflicto como algo natural a la propia ciencia. El análisis de las controversias científicas proporciona un marco de estudio sobre los complejos procesos del cambio científico. Su inclusión como categoría filosófica e histórica de análisis permite, pues, reducir la brecha que existía entre el estudio de la fase de descubrimiento y la fase de justificación de los conocimientos producidos (BRAGA; GUERRA; REIS, 2012).

Las comunidades científicas intentan resolver las controversias apelando a factores epistémicos (e.g., evidencias e inferencias) que, dentro de lo posible, no estén distorsionados por creencias personales, ideológicas, políticas y religiosas de los participantes (factores no-epistémicos). Sin embargo, historiadores, filósofos y sociólogos de la ciencia encuentran la presencia de tales aspectos no-epistémicos en la formación del juicio de una comunidad científica. La ciencia es una actividad cultural, que responde a necesidades, intereses, problemas sociales, políticos, económicos e ideológicos; y en toda controversia científica influyen factores tanto epistémicos como no-epistémicos (ACEVEDO-DÍAZ, 2006). Por tanto, la educación científica debe tener en cuenta ambos tipos.

Tolvanen et al. (2014) recomiendan centrarse solo en algunos aspectos de NDC, en lugar de plantear un debate general sobre NDC. La lectura de controversias científicas históricas, individualmente o en grupo pequeño, seguida de respuestas reflexionadas y razonadas a algunas cuestiones sobre aspectos de NDC, es un recurso útil para introducir tales aspectos en la educación científica (ACEVEDO-DÍAZ, 2008, 2009). Clough (2011) sugiere también que los relatos de HDC incluyan palabras de los científicos para resaltar la dimensión humana de la ciencia y añadir así autenticidad a las ideas de NDC que ilustran. Además, debe evitarse dar una visión mítica de los científicos (ALLCHIN, 2003), así como una interpretación anacrónica del pasado, que exagere la importancia de lo que ha contribuido a la ciencia actual, en vez de tratar de entender el contexto social del momento y los factores contingentes de su desarrollo. De lo contrario, se transmitirá una visión acumulativa y lineal del progreso de la ciencia (MONK; OSBORNE, 1997).

\section{Un caso de controversia de HDC para la formación del profesorado sobre NDC}

La enseñanza de la NDC tiene poca presencia en la educación científica de los diferentes niveles educativos españoles actualmente, sea cual sea el contexto y el enfoque que se considere. 
Si se opta por emplear un contexto histórico con un enfoque explícito y reflexivo, los profesores necesitan materiales curriculares adecuados para que sus estudiantes logren un aprendizaje eficaz de aspectos de NDC (MONK; OSBORNE, 1997; TOLVANEN et al., 2014). De acuerdo con ello, a continuación se muestra un relato de la controversia entre Pasteur y Pouchet sobre la generación espontánea (GE), preparado para la formación de estudiantes de profesorado de ciencias de Educación Secundaria (EPES). Esta controversia ha sido utilizada antes en España en la formación de EPES, con resultados moderadamente aceptables, para intentar superar la imagen simplista de la actividad científica (IZQUIERDO, 2008; VALLVERDÚ; IZQUIERDO, 2010). Sin embargo, hay que advertir que el trabajo de estos autores se hizo con una versión de la controversia mucho más simplificada que la que planteamos aquí, y se centró en otros objetivos. En nuestro caso, consideramos que si los EPES tienen una experiencia satisfactoria con la actividad como estudiantes, aumentarán las posibilidades de que luego la implementen en sus clases de ciencia con las adaptaciones precisas, aunque manteniendo fidelidad al texto que se propone aquí.

Se ha procurado evitar una visión sesgada a los aspectos experimentales en el texto histórico, intentando que el relato tenga el equilibrio necesario entre las perspectivas epistémica y no-epistémica (ACEVEDO-DÍAZ, 2006). De otra forma, en la narración se presta atención a los aspectos epistemológicos y contextuales, internos y externos a la comunidad científica, tal y como se recomienda hoy para enseñar NDC en la educación científica de una forma más holística (ACEVEDO-DÍAZ; GARCÍA-CARMONA, 2016a). Para ello, se han consultado diversas fuentes a la hora de elaborar el texto de la controversia: Collins y Pinch (1996), Dubos (1984), Farley y Geison (1994), Latour (1991), Martins (2009), Martins y Martins (1989), Raynaud (1999), Vallverdú e Izquierdo (2010) y Waller (2002).

\section{La controversia entre Pasteur y Pouchet sobre la generación espontánea}

\section{Antecedentes históricos}

Durante siglos se ha creído que, en circunstancias favorables, podían surgir seres vivos de mayor o menor tamaño en un recinto cerrado sin la intervención de progenitores semejantes a ellos; e.g., Aristóteles en la antigua Grecia, entre otros muchos. Esta teoría se conoce como generación espontánea (GE). A partir del siglo XVII, se informaba periódicamente de experimentos favorables y contrarios a la GE; e.g., Jean Babtiste van Helmont (1577-1644) a favor y Francisco Redi (1626-1697) en contra. La creencia en la GE se revitalizó posteriormente con el empleo del microscopio, atribuyéndose a seres microscópicos nuevos, que podían verse proliferar en pocas horas con ese instrumento. Las polémicas continuaron ahora acerca del origen de estos microorganismos en caldos de cultivo, e.g., John Needham (1713-1781) a favor de la GE y Lázaro Spallanzani (1729-1799) en contra. La GE también fue apoyada por naturalistas ilustres del siglo XVIII, como George Louis Leclerc, conde de Buffon (1707-1788), que sugirió en 1778 dos formas de GE en la historia de la Tierra: una para producir las criaturas que vivieron en las condiciones iniciales calientes del planeta, y otra para dar lugar a los antepasados de las formas modernas. 
A principios del siglo XIX, Jean Baptiste Lamarck (1744-1829) defendió la GE de organismos primitivos a partir de los cuales supuso que comenzaron las distintas líneas evolutivas (teoría transformista). Se produjo, al respecto, una larga polémica entre Isidore Geoffroy Saint-Hilaire (1805-1861), favorable a la GE asociada al transformismo, y George Cuvier (17691832), contrario a esta. En esta controversia, Cuvier mezcló sus argumentos científicos contra Geoffroy con aspectos religiosos, filosóficos y políticos para conseguir su propósito. Por ejemplo, asoció a sus oponentes con la Nathurphilosophie germana (Prusia era enemiga de Francia), pese a que Geoffroy negase numerosas veces esta asociación, y también los relacionó con el materialismo de los filósofos e ideólogos franceses de finales del siglo XVIII, a quienes se hacía responsables del régimen de terror durante la Revolución Francesa (FARLEY; GEISON, 1994).

A mediados del siglo XIX se sabía que las infusiones orgánicas susceptibles de putrefacción, en las que se había eliminado cualquier posibilidad de vida por una exposición prolongada a altas temperaturas, permanecían inalteradas a menudo y, por lo general, no se desarrollaba vida microscópica en ellas mientras estuvieran protegidas del contacto del aire. La entrada de aire hacía que los líquidos fermentaran o se pudrieran, apareciendo a los pocos días una gran variedad de microorganismos. ¿Se debía esto a que el aire era un factor esencial para la generación de vida, o a que era el medio a través del cual se introducían los gérmenes vivos? Los partidarios de la GE consideraban que el aire era necesario para la GE. Sus adversarios creían, en cambio, que el aire tan solo era el vector que introducía los gérmenes vivos de la putrefacción y la fermentación en los líquidos orgánicos.

Félix Archimède Pouchet (1800-1872) estaba convencido de que sus experimentos evidenciaban que la GE existía; de lo contrario, ¿cómo era posible que, tomando las debidas precauciones para evitar la entrada de cualquier germen en los frascos, siempre aparecieran seres vivos en las infusiones de heno al cabo de unos días? Hacia 1858, el estudio de los gérmenes y su papel en las fermentaciones, en las que estaba investigando desde 1855 (ACEVEDO-DÍAZ; GARCÍA-CARMONA, 2016c), llevó a Louis Pasteur (1822-1895) a interesarse por este asunto y entrar en el debate sobre la GE; una polémica en la que intervenían científicos, filósofos y teólogos. Biot y Dumas trataron de disuadirlo, pero Pasteur consideraba esta cuestión de gran importancia porque creía que le serviría para complementar sus estudios sobre las fermentaciones.

Cuando comenzó el debate entre ambos sobre la GE, Pasteur, químico y académico en París, tenía 37 años. Pouchet tenía casi 60 años; era médico y naturalista con gran experiencia en biología animal tradicional, director del Museo de Historia Natural de Rouen, académico y miembro honorario de varias sociedades científicas fuera de Francia. La controversia se extendió durante el periodo que va de 1859 a 1864.

\section{Inicio de la controversia}

El 20 de diciembre de 1858, Pouchet envió una detallada comunicación a la Academia de Ciencias de Francia (Academia, en adelante) anunciando la GE de microorganismos, bajo condiciones cuidadosamente controladas, en infusiones sometidas a altas temperaturas, que estaban en contacto con oxígeno producido químicamente para evitar la introducción de microorganismos que pudieran estar en el aire atmosférico. Pese a la sorpresa inicial de los académicos, muchos protestaron con vehemencia, entre ellos el influyente Claude Bernard. Rechazaron el 
descubrimiento sin siquiera repetir el experimento, suponiendo que los microorganismos se habían introducido accidentalmente en los medios de cultivo, o que el calor del horno aplicado a la infusión no había sido suficiente para matar los que hubiera.

El experimento de Pouchet consistía en tomar una botella con agua hirviendo, cerrada herméticamente, y sumergirla invertida en una vasija de mercurio. Cuando el agua se había enfriado, abría la botella bajo el mercurio e introducía medio litro de oxígeno y una pequeña cantidad de infusión de heno, calentada previamente a temperatura elevada en un horno durante bastante tiempo. Suponía que estas precauciones eran suficientes para impedir la presencia de microorganismos en la botella, pero estos siempre aparecían en la infusión de heno a los pocos días. El que los cultivos se contaminasen era una prueba experimental de la GE para Pouchet.

El debate entre Pasteur y Pouchet comenzó con mucha cortesía en el ámbito privado, como lo acredita la carta que Pasteur envió a Pouchet en febrero de 1859 (véanse extractos de esta en FARLEY; GEISON, 1994, p. 240-241 y en LATOUR, 1991, p. 485), a diferencia de la mala recepción que había tenido la comunicación de Pouchet entre los miembros de la Academia. El experimento parecía correcto y Pasteur lo aceptó en principio. Pero, poco después, pensó que el mercurio empleado en el momento de la entrada del aire en las botellas calentadas siempre contenía polvo y un gran número de microorganismos en su superficie. Pasteur eliminó la fuente de contaminación de los líquidos y del aire utilizando matraces con forma de balón y cuello de cisne. El uso de estos matraces especiales le fue sugerido a Pasteur por Balard, que había sido profesor suyo. Debido a las curvaturas del cuello y a que sus paredes estaban húmedas, las partículas de polvo quedaban atrapadas y no llegaban desde el exterior hasta el líquido aunque el aire circulase libremente. De este modo, la infusión podía permanecer sin microorganismos por un tiempo indefinido, como lo confirma que algunos de estos matraces aún se conservan inalterados en el Instituto Pasteur de París. Pasteur también diseñó un experimento para mostrar indirectamente la existencia de microorganismos en el aire y filtrarlos. Además, las observaciones de las muestras del aire filtrado en diferentes lugares le permitieron mostrar que existían grandes diferencias entre la cantidad y el tipo de microorganismos que aparecían, dependiendo del grado de contaminación de las muestras. Asimismo, preparó diversos experimentos para averiguar cuál era la menor cantidad de aire necesaria para contaminar los cultivos, lo que le sirvió de ayuda en el desarrollo de su teoría germinal.

Pasteur conjeturó que el aire puro sin calentar sería incapaz de producir la alteración de los líquidos orgánicos, si no tiene los gérmenes vivos de la fermentación, y que la cantidad de microorganismos que se encuentran en el aire variaría con la altitud. Hizo experimentos en el campo a poca altitud, en una de las montañas del macizo del Jura (a $850 \mathrm{~m}$ de altura) y en el glaciar La mer de glace (Montenvers, en los Alpes franceses, a $2000 \mathrm{~m}$ de altura). Encontró que los gérmenes aéreos son más abundantes en los lugares bajos, sobre todo cerca de las tierras cultivadas; y que hay menos cuando el aire permanece tranquilo durante largos periodos de tiempo, como en los sótanos y en las montañas, lejos de tierras habitadas o cultivadas. De los veinte matraces que abrió en el campo se alteraron ocho, cinco de los veinte abiertos en el Jura, y uno solo de los otros veinte desprecintados en La mer de glace. Pasteur hizo estos experimentos indirectos porque no era posible observar directamente si había o no microorganismos en el aire que entraba en los matraces. 


\section{Papel de la Academia: el premio Alhumpert}

Siguiendo una tradición habitual para fomentar la investigación, la Academia instituyó el 30 de enero de 1860 el premio Alhumpert, bajo el lema "Para arrojar, mediante experimentos cuidadosamente dirigidos, nueva luz sobre la cuestión de la llamada generación espontánea". Los experimentos de los dos investigadores se repetirían ante una comisión, que daría su dictamen sobre las pruebas presentadas. Según Farley y Geison (1994), esta comisión quedó constituida por una lista de miembros contrarios a la GE.

Pasteur y Pouchet se inscribieron para competir por el premio. Sin embargo, Pouchet y sus colegas Joly y Musset no estaban interesados en repetir las experiencias de Pasteur usando las infusiones de heno; querían hacer investigaciones diferentes. Si lo hubiera hecho, habría puesto en serios apuros a Pasteur, que había utilizado infusión de levadura como material putrescible en sus experimentos, mientras que Pouchet había usado infusión de heno. La esterilización por calor de la infusión de levadura puede conseguirse a los $100{ }^{\circ} \mathrm{C}$, mientras que se necesitan más de $120^{\circ} \mathrm{C}$ para eliminar todos los gérmenes de la infusión de heno. El tratamiento mediante calor aplicado por Pasteur $\left(100^{\circ} \mathrm{C}\right)$ habría sido insuficiente para eliminar los gérmenes del caldo de heno.

La comisión no aceptó el extenso programa de investigación de Pouchet, porque no creía que así pudieran llegar a resultados claros. Pouchet y sus colegas no aceptaron estas condiciones y se retiraron del concurso como protesta. Además, parece ser que algunos miembros habían anunciado su decisión favorable a Pasteur antes, incluso, de la presentación pública de los trabajos (FARLEY; GEISON, 1994), lo que irritó profundamente a Pouchet. Pasteur y sus colaboradores mostraron sus resultados experimentales, prestando especial atención a los aspectos técnicos con rigor y éxito. La Academia concedió el premio Alhumpert a Pasteur, en 1862, por su Mémoire sur les corpuscules organisés qui existent dans l'atmosphère (1861).

Sin embargo, Pouchet y sus colegas Joly y Musset no se desanimaron. En 1863, Pouchet publicó una nueva edición aumentada de su libro, reiterando su creencia en la GE. Ese mismo año comunicó que, junto a sus otros dos colegas, había intentado reproducir los hallazgos de Pasteur sobre la distribución de los gérmenes en el aire con resultados distintos. Realizaron sus experimentos a diferentes altitudes en los Pirineos de Aragón, llegando hasta la Maladeta (3.000 m de altura). Encontraron que dondequiera que se recogía un volumen notable de aire que entraba en contacto con un líquido orgánico, al abrir el matraz herméticamente sellado, el líquido mostraba el desarrollo de gérmenes vivos. Cuatro matraces se rompieron de los doce que llevaban. Los cuatro que desprecintaron en Rencluse (2083 m de altura) se contaminaron, así como los otros cuatro que abrieron en la Maladetta.

Con el fin de dilucidar la cuestión, Pouchet pidió a la Academia que volviera a nombrar una comisión para repetir ante ella los experimentos que los dos grupos de investigadores habían hecho con resultados tan diferentes. Sin embargo, esta nueva comisión también se constituyó sesgada en contra de la GE. Tres de sus cinco miembros ya habían estado en la comisión anterior. Uno de los nuevos era Balard, que había sugerido a Pasteur el diseño de los matraces con cuello de cisne, y el otro también era partidario de Pasteur (FARLEY; GEISON, 1994).

Pasteur aceptó el reto, y después de un aplazamiento solicitado por Pouchet, así como de múltiples discusiones sobre la programación de las experiencias, Pouchet, Joly y Musset aceptaron asistir a la cita del 22 de junio de 1864, en el Museo de Historia Natural de París. Ese 
día, los dos grupos debían presentar sus trabajos de acuerdo con un programa organizado por la comisión. Pasteur llegó con sus colaboradores listo para la prueba, pero Pouchet y sus colegas tampoco se presentaron esta vez ${ }^{4}$. El fallo de la comisión a favor de Pasteur, dictaminado el 20 de febrero de 1865, fue cuidadosamente enunciado y referido estrictamente a los resultados, sin involucrar a los aspectos teóricos más generales de la GE.

La actitud tan desigual con Pasteur y Pouchet de las dos comisiones nombradas por la Academia nos lleva a incluir la influencia de los aspectos sociológicos internos a la comunidad científica. Según varios autores (e.g., FARLEY; GEISON, 1994; LATOUR, 1991), todos los miembros de ambas comisiones de la Academia eran favorables a Pasteur y, por tanto, Pouchet consideró que no se le daba una oportunidad equitativa para exponer sus ideas y sus experimentos. Sin embargo, Raynaud (1999) sostiene que la correspondencia disponible entre Pouchet y los miembros de la Academia muestra claramente que este tenía buena relación con muchos de ellos, incluido alguno de los comisionados, y que las dos comisiones, constituidas en $1861 \mathrm{y}$ 1864, no se confabularon a favor de Pasteur.

\section{Aspectos contextuales}

Pouchet y Pasteur realizaron sus experimentos sobre la GE cuando estaba en auge la disputa relativa al darwinismo. Clémence Royer había traducido al francés El origen de las especies de Darwin, en 1862, incluyendo un vehemente prefacio a favor del materialismo, el ateísmo y la República. La polémica sobre la GE se vio así ligada a la de la evolución inevitablemente. Hablar de Darwin o de GE suponía referirse al mismo tiempo a biología, aspectos sociales, Dios y formas de gobierno ${ }^{5}$ (LATOUR, 1991). Pasteur era un conservador fiel al emperador Luis Napoleón (Napoleón III) ${ }^{6}$, el cual a su vez le tuvo en gran estima ${ }^{7}$ (FARLEY; GEISON, 1994). Aunque Pouchet no era un materialista radical (FARLEY; GEISON, 1994), sino un conservador moderado (LATOUR, 1991), la burguesía católica parisina recelaba de sus ideas. Pouchet había desarrollado su teoría de la GE antes de que estallara en Francia la polémica darwinista, con fuertes implicaciones religiosas y políticas. Después, se mantuvo siempre fiel a su teoría, pese a ser consciente de que podía suponer una amenaza a las creencias políticas y religiosas que el mismo compartía (FARLEY; GEISON, 1994).

\footnotetext{
${ }^{4}$ El programa organizado por la comisión de la Academia comenzaba con la exposición de Pasteur. Además, le permitía utilizar sus resultados anteriores, obtenidos en La mer de glace (Montenvers), en vez de presentar los nuevos resultados. Pouchet y sus colegas desistieron de presentarse de nuevo como protesta a lo que consideraron una actitud sesgada de la Academia en contra de ellos (MARTINS, 2009).

${ }^{5}$ Diversos regímenes políticos de signo muy diferentes se sucedieron en Francia durante el siglo XIX: Primer Imperio Napoleónico (1799), Restauración de la Monarquía, con varios monarcas (1815), Segunda República (1848), Segundo Imperio (1852) y Tercera República (1870), que incluye un brevísimo período de gobierno popular durante poco más de dos meses conocido como La Comuna (marzo-mayo de 1871).

${ }^{6}$ En diciembre de 1851, Luis Napoleón disolvió la Segunda República, de la que era Presidente desde 1848. Después del golpe de estado, se autoproclamó Emperador, en 1852, con el inestimable apoyo de la Iglesia Católica y la burguesía conservadora.

${ }^{7}$ Napoleón III alentó las investigaciones más prácticas de Pasteur y le ayudó a conseguir recursos económicos para continuarlas. Le nombró Caballero de la Orden Imperial de la Legión de Honor (1853) y, años después, Comandante de la misma Orden (1868).
} 
Como señala Gálvez (1988), un examen de los escritos de Pouchet muestra que sus creencias religiosas influyeron en su trabajo al menos tanto como las de Pasteur. Cuando publicó su libro Hétérogénie ou traité de la génération spontanée, en 1859, criticó el materialismo y el darwinismo. Según el propio Pouchet, la negación de la GE equivalía a adoptar una posición atea y caer en el darwinismo. Para él, era necesaria la intervención divina en la creación hasta la actualidad para reconstruir las especies después de cada catástrofe geológica; esto es, para aceptar la GE mediante la heterogénesis (el nacimiento de seres vivos a partir de sustancias orgánicas procedentes de un progenitor que no es semejante) y no por abiogénesis (generación de organismos vivos a partir de la materia inanimada). Sin embargo, la GE también servía de argumento a los ateos para prescindir de Dios, pues la materia podía engendrar seres vivos por sí sola. De este modo, Pasteur asoció la posición de Pouchet con el materialismo, el ateísmo y el darwinismo en su célebre conferencia impartida, el 7 de abril de 1864, en las "Veladas científicas de la Sorbona" ${ }^{8}$. No obstante, una vez hecho esto, afirmó:

\begin{abstract}
No hay aquí ni religión, ni filosofía, ni ateísmo, ni materialismo, ni espiritualismo que valga [...] Es una cuestión de hecho; la he abordado sin ideas preconcebidas, tan dispuesto a declarar, si la experiencia me hubiera impuesto la confesión, que existían generaciones espontáneas, como estoy convencido hoy de que los que lo afirman tiene una venda sobre los ojos. (PASTEUR, 1944).
\end{abstract}

Latour (1991) señala que invocar a Dios ante ese auditorio habría sido negativo; pero recurrir a ello para, inmediatamente, volver a situar el foco de la atención en los experimentos y los hechos, garantizaba la repercusión de los argumentos de Pasteur y debilitaba los de su adversario. Pasteur mostró así su habilidad en el uso de la comunicación pública. Ahora bien, Pouchet coincidía con Pasteur en que los resultados experimentales obtenidos debían ser el árbitro inapelable, fueran cuales fueran las grandes cuestiones que se cernían sobre la GE. Se puede recurrir a Dios, al soberano, la moral o la herencia, pero es imprescindible comprobar si el líquido de los matraces se enturbia o sigue inalterado. Los factores contextuales pueden condicionar la controversia, pero son incapaces de enturbiar los matraces. En consecuencia, Pasteur puso todo su empeño durante la conferencia en mostrar los fallos de los experimentos de Pouchet, para intentar desmontar así la teoría de la GE (véanse, e.g., FARLEY; GEISON, 1994, p. 251-254; LATOUR, 1991, p. 479-481, 487-488, 496).

\title{
Resurgimiento y decaimiento de la controversia
}

Aunque la Academia protegió a Pasteur, dando por cerrada la controversia y sin proponer nuevas investigaciones, los oponentes no permanecieron callados. La polémica de la GE se reabrió con fuerza en Inglaterra, durante 1872, a partir de los experimentos realizados por el médico Henry C. Bastian, publicados en su voluminoso libro The beginning of life; being some

\footnotetext{
${ }^{8}$ Véase una traducción al castellano de la conferencia en: < http://www.valencia.edu/ orilife/textos/Pasteur. pdf>. Visitado el: 30 ago. 2016.
} 
account of the nature, modes of origin and transformation of lower organisms. Pasteur tuvo que hacer nuevas investigaciones, comprobando que si no se alcanzaba una temperatura de $120^{\circ} \mathrm{C}$, no había seguridad de matar a todos los microorganismos. Se vio obligado a reconocer las limitaciones de las técnicas experimentales que había usado en su controversia con Pouchet, y diseñó procedimientos mucho más precisos para solventarlo. Ello contribuyó también a mejorar el desarrollo de la asepsia clínica en todas las especialidades quirúrgicas (DUBOS, 1984).

Más adelante, en su libro Essays on the floating matter of the air in relation to putrefaction and infection (1881), el físico británico John Tyndall (1820-1893) expuso una demostración concluyente de que la putrefacción no se produce en presencia de aire libre de polvo (DUBOS, 1984). Preparó cámaras experimentales con la superficie interior recubierta de glicerina. Las cámaras cerradas (cajones transparentes) se dejaban sin tocar durante varios días hasta que un rayo de luz, al pasar por unas ventanas laterales, indicaba que toda la materia flotante del aire se había sedimentado y había quedado adherida a las superficies de glicerina. Bajo estas condiciones, se podían exponer al aire de la cámara toda clase de líquidos orgánicos esterilizados, orina, caldo e infusiones vegetales que permanecían sin alterarse durante meses. Por tanto, el aire "ópticamente vacío", como lo llamó Tyndall, era estéril.

\section{Cuestiones de NDC relativas a la controversia entre Pasteur y Pouchet sobre la generación espontánea}

Como orientación para el formador de EPES, a continuación se hacen algunos comentarios sobre las cuestiones de NDC relacionadas con la controversia científica que se proponen en el Cuadro 1.

Cuadro 1. Cuestiones propuestas para reflexionar a partir de la lectura

C.1. ¿Cuál crees que fue el papel de las creencias teóricas en la interpretación de las observaciones? ¿Por qué?

C.2. ¿Qué papel crees que tuvieron los diseños experimentales en los resultados obtenidos? ¿Por qué?

C.3. ¿Crees que la controversia se cerró mediante un experimento crucial? ¿Por qué?

C.4. ¿Crees que hubo subjetividad en la controversia? ¿Por qué?

C.5. ¿Crees que el procedimiento que empleó la comunidad científica para juzgar la controversia evitó la subjetividad? ¿Por qué?

C.6. ¿Crees que influyó la religión en la controversia? ¿Por qué?

C.7. ¿Crees que influyó la política en la controversia? ¿Por qué?

C.8. ¿Qué procedimientos crees que se usaron para comunicar los resultados de las investigaciones a sus colegas y al público interesado? Coméntalos.

C.9. ¿Qué factores crees que influyeron más en la resolución de la controversia? ¿Por qué?

Fuente: elaborado por los autores. 


\section{C.1. ¿Cuál crees que fue el papel de las creencias teóricas en la interpretación de las observaciones? ¿Por qué?}

Pouchet creía en su teoría de la GE, la heterogénesis, que permitía explicar discontinuidades y rupturas en las formas de vida después de las catástrofes geológicas; mientras que la posición de Pasteur era contraria a la GE. Pouchet y sus seguidores interpretaban la aparición de microorganismos en las infusiones, dentro de aire aparentemente puro, como una prueba de la GE. Si los microorganismos no aparecían, el resultado era debido a un fallo experimental; e.g., que las propiedades vitales del aire se habían destruido en el proceso de purificación. Por el contrario, para Pasteur y los que no creían en la GE, la aparición de microorganismos en las infusiones, dentro de aire supuestamente puro, se interpretaba como un error experimental que había pasado inadvertido; e.g., que la infusión o el aire que entraba se habían contaminado accidentalmente. Si los microorganismos no aparecían, era una prueba de que la GE no existía (COLLINS; PINCH, 1996; VALLVERDÚ; IZQUIERDO, 2010).

\section{C.2. ¿Qué papel crees que tuvieron los diseños experimentales en los resultados obtenidos? ¿Por qué?}

Tanto Pouchet como Pasteur intentaron diseñar experimentos cuidadosamente y con el mayor rigor posible. Sin embargo, emplearon infusiones diferentes, que no intercambiaron, y las utilizaron en función de sus conclusiones. Como se ha señalado antes, ambos interpretaban de manera diferente los resultados erróneos que obtenían a veces. Una cosa son las observaciones y otra las inferencias que se hacen para explicarlas.

\section{C.3. ¿Crees que la controversia se cerró mediante un experimento crucial? ¿Por qué?}

Los resultados de la controversia entre Pasteur y Pouchet son más dudosos de como suelen aparecer en muchos libros de texto y relatos hagiográficos, que presentan a Pasteur como vencedor en su polémica sobre la GE. No suele haber experimentos cruciales que cierren las controversias por completo. Como en otras ocasiones de HDC, lo que en un momento dado se consideró definitivo es posible que cambie en el futuro con experimentos más refinados y, sobre todo, cuando se contempla desde otra perspectiva.

¿Cuál fue entonces el alcance del trabajo de Pasteur sobre la GE? La difusión pública de sus resultados puso en entredicho la GE continua; es decir, la relativa a la producción continuada a través del tiempo y circunscrita a unos pocos seres vivos, que nacen siempre y constantemente según el mismo mecanismo. Pasteur sentó las bases para señalar con pocas dudas que lo viviente nace de lo viviente, pero no explicó el origen de lo viviente, que podría entenderse mediante la GE en un solo acto, o unos pocos, en el pasado remoto. De este modo, una de las consecuencias de los trabajos de Pasteur fue el crecimiento de las concepciones vitalistas, teleológicas y animistas. Por ello, Ernst Haeckel (1834-1919) declaró que negar la GE significaba aceptar que la vida apareció espontáneamente siguiendo las leyes de las propiedades fisicoquímicas de la materia, o bien que fue producida por una creación sobrenatural divina.

\section{C.4. ¿Crees que hubo subjetividad en la controversia? ¿Por qué?}

Los resultados experimentales fueron favorables y contrarios a la GE durante el periodo de la controversia, por lo que era muy difícil tomar una decisión objetiva. Las interpretaciones 
de las observaciones de ambos científicos estaban influidas fuertemente por sus creencias; e.g., sobre la naturaleza de la vida, la naturaleza de la putrefacción y la fermentación, etc. Sus creencias teológicas, sociales y políticas también pudieron tener importancia.

\section{C.5. ¿Crees que el procedimiento que empleó la comunidad científica para juzgar la controversia evitó la subjetividad? ¿Por qué?}

La forma de resolver una polémica científica en Francia, circa 1840, solía ser mediante la creación de una comisión de pares académicos para revisar los resultados obtenidos por los contendientes. Tal fue el caso de la constitución de dos comisiones por la Academia de Ciencias, en 1861 y 1864, para dirimir sobre los resultados de Pasteur y Pouchet, y otorgar el premio Alhumpert al vencedor. Los hagiógrafos de Pasteur han obviado el contexto de este asunto por lo general. Sin embargo, otros historiadores externalistas de la ciencia y sociólogos de la ciencia de diversas corrientes relativistas han enfatizado el contexto de la controversia a partir de la obra de Pennetier, un discípulo de Pouchet. Estos autores insisten en que hubo una confabulación de la Academia contra Pouchet, resaltando que ambas comisiones estaban sesgadas desde su constitución a favor de Pasteur. No obstante, el estudio de la correspondencia privada de Pouchet con miembros de la Academia, incluyendo dos de los comisionados por esta para resolver la controversia, muestra que este mantenía una buena amistad con ellos (RAYNAUD, 1999). Valga este comentario como prevención de posibles sesgos al establecer conclusiones cuando las fuentes de HDC son de un solo signo o similar.

\section{C.6. ¿Crees que influyó la religión en la controversia? ¿Por qué?}

McComas y Kampourakis (2015) afirman que las motivaciones de Pasteur no eran exclusivamente científicas. Según estos autores, Pasteur era un católico conservador dispuesto a contrarrestar los argumentos del materialista radical Pouchet ${ }^{9}$. Así, Pasteur no habría tenido como objetivo principal descubrir lo que pasaba en los matraces, sino confirmar una conclusión a la que ya habría llegado antes subjetivamente por motivos no científicos. Es cierto que, en la famosa conferencia de la Sorbona, Pasteur dijo que la GE podría servir de argumento a los ateos para prescindir de Dios. Sin embargo, Pouchet también sostuvo, en su libro Heterogénesis, que negar la GE equivalía a adoptar una posición atea y caer en el darwinismo. Pasteur era un devoto católico y Pouchet miembro de una familia protestante ${ }^{10}$, que estaba familiarizado con las Escrituras Sagradas. Asimismo, Pouchet era contrario al darwinismo ${ }^{11}$ y al transformismo. Los aspectos teológicos condicionaron la polémica entre Pasteur y Pouchet, pero no tenían la capacidad de enturbiar los matraces.

\section{C.7. ¿Crees que influyó la política en la controversia? ¿Por qué?}

La respuesta a la pregunta ¿somos o no iguales que nuestros padres? tenía fuertes repercusiones sociales y políticas en aquella época. Una respuesta afirmativa se asociaba a una

\footnotetext{
${ }^{9}$ Pouchet no era un materialista radical (FARLEY; GEISON, 1994; GÁLVEZ, 1988). No creemos que sea una buena praxis de HDC la que, para derribar mitos, genera otros accidental o intencionadamente.

${ }^{10}$ Latour (1991, p. 488) afirma erróneamente que Pouchet era un buen católico.

${ }^{11}$ Vallverdú e Izquierdo (2010, p. 58) califican erróneamente a Pouchet de darwinista.
} 
posición conservadora, que servía de argumento para mantener el estatus social; en cambio, negarlo se vinculaba a una postura revolucionaria justo por lo contrario (LATOUR, 1991). El incendiario prefacio de Clémence Royer en la edición francesa de 1862 de El origen de las especies lo deja bien claro. El régimen político de Francia era absolutista durante el periodo de la polémica (el Segundo Imperio de Napoleón III), y los convulsos movimientos políticos de la época influyeron públicamente, de alguna manera, en la controversia Pasteur vs. Pouchet; pero ambos tenían ideas políticas conservadoras ${ }^{12}$, si bien las de Pasteur eran más firmes y comprometidas con el poder institucionalizado que las de Pouchet.

\section{C.8. ¿Qué procedimientos crees que se usaron para comunicar los resultados de las investigaciones a sus colegas y al público interesado? Coméntalos.}

Memorias académicas y libros eran los medios habituales para publicar en la época. Asimismo, las ideas y resultados se anticipaban y comentaban con los colegas en cartas (RAYNAUD, 1999). A veces también se impartían conferencias ante un auditorio numeroso, que luego la prensa trasladaba al público en general; e.g., la conferencia de Pasteur del 7 de abril de 1864 en el anfiteatro de la Sorbona. Por tanto, tiene especial interés el acceso de los científicos a los medios de comunicación. Según Izquierdo (2008), Pasteur tuvo la oportunidad de exponer sus ideas en conferencias a las que asistió mucho público en París, mientras que Pouchet no. Pasteur manejaba con eficacia la comunicación científica, con una excelente retórica y una puesta en escena histriónica, como ocurrió en la mencionada conferencia de las "Veladas científicas de la Sorbona". No obstante, Pouchet también movilizó a la prensa, sobre todo en 1864, para contrarrestar públicamente cualquier evaluación académica de sus resultados experimentales. Pouchet tenía contacto con periodistas amigos, que escribían a favor de la GE en el debate público paralelo que se realizaba en la prensa. El propio Pouchet entregaba a veces a los periodistas lo que quería ver publicado. Así pues, Pouchet estaba convencido del equilibrio entre ambos foros del debate, el académico y el periodístico, como lo atestigua una carta de junio de 1864 que envió a Noël, uno de los periodistas de su círculo de amistades: "En compensación, creo que la opinión pública es muy favorable a nosotros, y la conferencia infame de Paracelso $\mathrm{II}^{13}$ ha tenido un efecto deplorable en todas partes." (RAYNAUD, 1999, p. 293, traducción nuestra).

\section{C.9. ¿Qué factores crees que influyeron más en la resolución de la controversia? ¿Por qué?}

Las decisiones de los científicos para apoyar o rechazar una teoría no se basan en las pruebas empíricas exclusivamente. Los motivos para que los resultados de Pasteur se aceptaran frente a los de Pouchet son muy diversos, y es difícil saber cuáles influyeron más. Cuando la ciencia está en construcción, los factores no-epistémicos pueden tener tanto o más peso que los epistémicos. La controversia entre Pasteur y Pouchet está impregnada de conflictos entre ambos aspectos.

El enfoque externalista de la HDC y la Sociología de la Ciencia relativista han dado una interpretación de la controversia entre Pasteur y Pouchet sobre la GE, que sugiere el éxito del primero por la influencia en gran parte de factores religiosos, políticos, sociales y de cabildeo,

\footnotetext{
${ }^{12}$ Vallverdú e Izquierdo (2010, p. 58) afirman erróneamente que Pouchet tenía ideas políticas progresistas.

${ }^{13}$ Paracelso II era el mote que Pouchet y sus colaboradores le habían puesto a Pasteur (LATOUR, 1991, p. 489). 
todos ellos aspectos no-epistémicos, en vez de por la evidencia experimental. La controversia supuso mucho más que los experimentos, por supuesto, y los factores contextuales influyeron, pero ¿hasta qué punto? Según Raynaud (1999), un historiador de la ciencia de tendencia racionalista, la derrota de Pouchet se debió a la combinación de un conjunto de factores, tales como sus retiradas ante ambas comisiones de la Academia, su fe dogmática en la heterogénesis, la presión que ejerció sobre la prensa, que fue mal vista por la Academia, etc. Para Gálvez (1988), el motivo principal fue que el fuerte compromiso de Pouchet con la teoría de la GE no le permitía abrirse a modificaciones a la luz de las nuevas evidencias experimentales.

Relacionado con esta cuestión, cabe destacar también la controversia entre los investigadores racionalistas y relativistas respecto a la reflexión histórica acerca de la controversia entre Pasteur y Pouchet sobre la GE; es decir, la existencia de una meta-controversia sobre la propia controversia científica. Esto es consecuencia, sin duda, de la multitud de factores que influyen en la aceptación de las teorías científicas, así como de la dificultad que entraña un análisis completo de todos los aspectos por las diferentes interpretaciones puestas en juego.

\section{Recomendaciones metodológicas para la implementación educativa de la controversia}

Para finalizar, se proporcionan unas indicaciones metodológicas para la implementación de la controversia en la formación de EPES, siguiendo un proceso en tres fases.

Fase I: Lectura del texto de la controversia histórica y respuestas a las cuestiones. Puesto que el propósito general es incentivar la reflexión sobre la controversia presentada y, en particular, valorar las aportaciones de esta al aprendizaje de ciertos aspectos de NDC, se planteará a los EPES, organizados en equipos, su lectura sin mediar enseñanza previa. En el caso de que se opte por entregar el texto para una lectura individual previa fuera del aula, se puede pedir a los EPES un testigo de lectura en el que, por ejemplo, destaquen las ideas que les parezcan centrales en el texto, o cuáles son los aprendizajes que creen que se pueden conseguir en el aula con este material. De este modo, se dispondría de una información inicial individual, diferente de las repuestas a las cuestiones de NDC del cuadro 1, que también puede ser interesante. Esta pregunta se repetiría al final de la tercera fase, incorporándose las respuestas en el informe final.

Realizada la lectura, los equipos responderán a las cuestiones que se formulan en el cuadro 1. Las respuestas deben emanar de una discusión reflexiva y consensuada entre los miembros del equipo, y se registrarán en un informe. Si hubiera puntos de vista divergentes, que imposibilitaran consensuar una respuesta común, se pueden expresar las distintas posiciones razonadas ante una misma pregunta. Esta primera fase sirve para hacer explícitas las ideas sobre los aspectos de NDC planteados en las preguntas, y para su desarrollo en el aula se estima necesario un tiempo aproximado de 2 horas.

Fase II: Puesta en común de las respuestas de los equipos a las cuestiones formuladas. Tras responder a las cuestiones relativas al texto, los equipos compartirán y discutirán sus respuestas en clase durante una sesión en torno a 1,5 horas. El papel del formador en esta fase es moderar el debate entre los equipos e introducir aquellas aclaraciones, preguntas, etc. que lo enriquezcan todo lo posible. La intención es que lleguen a conclusiones comunes sobre los aspectos tratados de NDC, pero sin adoctrinamiento; es decir, sin tratar de imponer las visiones que pudieran ser 
más adecuadas. En este sentido, y ante posibles ideas de NDC que estén alejadas de las aceptadas actualmente, se procurará generar nuevos conflictos cognitivos para que los estudiantes de profesorado se replanteen libremente sus puntos de vista.

Cabe la posibilidad de que la moderación del debate se comparta entre el formador y un estudiante de profesorado. La razón es doble. Por una parte, la moderación de debates es una tarea que se supone que los EPES desempeñarán como docentes. Por otra, permite que el formador se distancie algo de la gestión del debate y pueda centrarse mejor en tomar notas de las intervenciones, tanto para reconducir la discusión en caso necesario como para la evaluación posterior.

Fase III: Conclusiones tras la puesta en común. Una vez que las respuestas a las cuestiones planteadas se hayan discutido en clase, cada equipo revisará sus respuestas iniciales para completar, matizar o reafirmar sus ideas y argumentos sobre los aspectos de NDC abordados. Todo ello se registrará en un informe final que se entregará con las conclusiones de equipo, a continuación de las respuestas iniciales que elaboraron.

\section{Consideraciones finales y perspectivas futuras}

Concluimos reiterándonos en que los episodios de HDC, y los de controversia en particular, son un recurso con gran potencial didáctico para el aprendizaje de la NDC. Pero, para ello, tal y como hemos indicado, las controversias deben adaptarse para su uso educativo, sintetizándolas del modo más fiel posible a hechos históricos contrastados y no sesgados, que permitan ilustrar algunos aspectos relevantes de la NDC, formulados explícitamente como cuestiones para reflexionar sobre la ciencia. Esto es lo que hemos pretendido justamente con la presentación de este trabajo sobre la controversia entre Pasteur y Pouchet acerca de la GE.

La controversia mostrada pertenece a un conjunto de textos de HDC y de Historia de la Tecnología, que hemos preparado como recursos educativos para la formación de EPES en algunos aspectos de NDC y de Naturaleza de la Tecnología (ACEVEDO-DÍAZ; GARCÍA-CARMONA, 2016b, 2016c; ACEVEDO-DÍAZ; GARCÍA-CARMONA; ARAGÓN, 2016). De este modo, será posible comprobar si las respuestas a cuestiones de NDC idénticas, o similares, surgen también en otros episodios de HDC y no se limitan solo a la controversia sobre la GE. Estos textos de HDC están siendo implementados como actividades de aula en el Máster de Formación del Profesorado de Ciencias de Educación Secundaria de dos universidades de España. Respecto a la controversia sobre la GE, en particular, los resultados que se obtengan servirán para analizar la validez de las cuestiones de NDC con relación al texto histórico, así como la metodología usada en la implementación, con vista a mejorar la eficacia de la propuesta en sucesivas implementaciones con otros EPES.

Asimismo, estamos trabajando con actividades relativas a la enseñanza de la NDC en la formación inicial del profesorado, usando como recurso educativo noticias de prensa sobre episodios de ciencia contemporáneos (e.g., GARCÍA-CARMONA, 2014, 2015; GARCÍA-CARMONA; ACEVEDO-DÍAZ, 2016). Ello permite complementar los episodios de ciencia del pasado con los actuales, tal y como recomienda Clough (2011), a fin de hacer las comparaciones pertinentes. En este sentido, se podría añadir una actividad, entre las fases de implementación I y II, orientada a identificar otros episodios de HDC, o de la ciencia de hoy, en los que se 
reconozcan situaciones controvertidas similares a la presentada en este trabajo, para su análisis reflexivo a partir de cuestiones como las del cuadro 1.

Por último, el proceso de enseñanza sobre aspectos de NDC podría complementarse con una actividad consistente en que los EPES consulten algunos de los libros de texto de Secundaria y Bachillerato más habituales, y comparen la información que ofrecen sobre la GE con la expuesta en el texto de la controversia presentado. Así, se les da la oportunidad de analizar las limitaciones de los libros de texto, en general, para tratar la ciencia escolar en sintonía con la HDC y, en concreto, para abordar explícitamente cuestiones de NDC similares a las del cuadro 1.

\section{Referencias}

ABD-EL-KHALICK, F. S. The influence of history of science courses on students' conceptions of the nature of science. 1998. Thesis (Ph. D. in Science Education) School of Education, Oregon State University, Oregon, 1998. Disponible en: <http://hdl. handle.net/1957/9728>. Visitado el: 30 ago. 2016.

. Teaching science with history. The Science Teacher, Arlington, v. 66, n. 9, p. 18-22, 1999.

. Teaching with and about nature of science, and science teacher knowledge domains. Science \& Education, Dordrecht, v. 22, n. 9, p. 2087-2107, 2013.

ABD-EL-KHALICK, F. S.; LEDERMAN, N. G. The influence of history of science course on students' views of nature of science. Journal of Research in Science Teaching, Malden, v. 37, n. 10, p. 1057-1095, 2000.

ACEVEDO-DÍAZ, J. A. Análisis de algunos criterios para diferenciar entre ciencia y tecnología. Enseñanza de las Ciencias, Barcelona, v. 16, n. 3, p. 409-420, 1998. Disponible en: < https://dialnet.unirioja.es/servlet/articulo?codigo=94932>. Visitado el: 30 ago. 2016.

. Enfoques explícitos versus implícitos en la enseñanza de la naturaleza de la ciencia.

Revista Eureka sobre Enseñanza y Divulgación de las Ciencias, Cádiz, v. 6, n. 3, p. 355386, 2009. Disponible en: < http://www.redalyc.org/pdf/920/92013010004.pdf >. Visitado el: 30 ago. 2016.

. El estado actual de la naturaleza de la ciencia en la didáctica de las ciencias. Revista

Eureka sobre Enseñanza y Divulgación de las Ciencias, Cádiz, v. 5, n. 2, p. 178-198, 2008. Disponible en: <http://www.redalyc.org/pdf/920/92050202.pdf>. Visitado el: 30 ago. 2016.

Relevancia de los factores no-epistémicos en la percepción pública de los asuntos tecnocientíficos. Revista Eureka sobre Enseñanza y Divulgación de las Ciencias, Cádiz, v. 3, n. 3, p. 369-390, 2006. Disponible en: <http://www.redalyc.org/pdf/920/92030303. pdf>. Visitado el: 30 ago. 2016. 
ACEVEDO-DÍAZ, J. A.; GARCÍA-CARMONA, A. "Algo antiguo, algo nuevo, algo prestado": tendencias sobre la naturaleza de la ciencia en la educación científica. Revista Eureka sobre Enseñanza y Divulgación de las Ciencias, Cádiz, v. 13, n. 1, p. 3-19, 2016a. Disponible en: <http://www.redalyc.org/articulo.oa?id=92043276002>. Visitado el: 30 ago. 2016.

. Una controversia de la historia de la tecnología para aprender sobre naturaleza de la tecnología: Tesla vs. Edison - la guerra de las corrientes. Enseñanza de las Ciencias, Barcelona, v. 34, n.1, p. 193-209, 2016b. Disponible en: <http://ensciencias.uab.es/article/ view/v34-n1-acevedo-garcia/1916-pdf-es>. Visitado el: 30 ago. 2016.

. Uso de la historia de la ciencia para comprender aspectos de la naturaleza de la ciencia: fundamentación de una propuesta basada en la controversia Pasteur versus Liebig sobre la fermentación. Revista Iberoamericana de Ciencia, Tecnología y Sociedad, Buenos Aires, v. 11, n. 33, , p. 203-226, 2016c. Disponible en: < http://www.revistacts.net/ files/Volumen_11_Numero_33/AcevedoEDITADOREVISADO.pdf > . Visitado el: 30 ago. 2016.

ACEVEDO-DÍAZ, J. A.; GARCÍA-CARMONA, A.; ARAGÓN, M. M. Un caso de historia de la ciencia para aprender naturaleza de la ciencia: Semmelweis y la fiebre puerperal. Revista Eureka sobre Enseñanza y Divulgación de las Ciencias, Cádiz, v. 13, n. 2, p. 408-422, 2016. Disponible en: <http://reuredc.uca.es/index.php/tavira/article/viewFile/921/886>. Visitado el: 30 ago. 2016.

ALLCHIN, D. Pseudohistory and pseudoscience. Science \& Education, Dordrecht, v. 13, n. 3, p. 179-195, 2004.

Scientific myth-conceptions. Science Education, Malden, v. 87, n. 3, p. 329-351, 2003. Disponible en: < http://www.tc.umn.edu/ allch001/papers/myth.pdf>. Visitado el: 30 ago. 2016.

BRAGA, M.; GUERRA, A.; REIS, J. C. The role of historical-philosophical controversies in teaching sciences: the debate between Biot and Ampère. Science $\boldsymbol{\&}$ Education, Dordrecht, v. 21, n. 6, p. 921-934, 2012.

CLOUGH, M. P. The story behind the science: bringing science and scientists to life in postsecondary science education. Science \& Education, Dordrecht, v. 20, n. 7-8, p. 701-717, 2011.

COLLINS, H. M.; PINCH, T. El gólem: lo que todos deberíamos saber acerca de la ciencia. Barcelona: Crítica, 1996.

CONFEDERACIÓN DE SOCIEDADES CIENTÍFICAS DE ESPAÑA. Informe

Enciende: enseñanza de las ciencias en la didáctica escolar para edades tempranas en España. Madrid: COSCE, 2011.

DUBOS, R. J. Pasteur. Barcelona: Salvat, 1984. 
ECHEVERRÍA, J. Ciencia y valores. Barcelona: Destino, 2002.

EUROPEAN COMISSION. Education, Audiovisual and Culture Executive Agency.

Eurydice. Science education in Europe: national policies, practices and research. Brussels, 2011. Disponible en: <https://webgate.ec.europa.eu/fpfis/mwikis/eurydice/index.php/ Publications:Science_Education_in_Europe:_National_Policies,_Practices_and_Research $>$. Visitado el: 31 ago. 2016.

FARLEY, J.; GEISON, G. L. Ciencia, política y generación espontánea en la Francia del siglo diecinueve: el debate Pasteur-Pouchet. In: SOLÍS, C. (Org.). Razones e intereses: la historia de la ciencia después de Kuhn. Barcelona: Paidós, 1994. p. 219-263.

FORATO, T. C. M.; MARTINS, R. A.; PIETROCOLA, M. A. Historiografia e natureza da ciência na sala de aula. Caderno Brasileiro de Ensino de Física, Florianópolis, v. 28, n. 1, p. 27-59, 2011.

GÁLVEZ, A. The role of the French Academy of Sciences in the clarification of the issue of spontaneous generation in the mid-nineteenth century. Annals of Science, Abingdon, v. 45, p. 345-365, 1988.

GARCÍA-CARMONA, A. Naturaleza de la ciencia en noticias científicas de la prensa: análisis del contenido y potencialidades didácticas. Enseñanza de las Ciencias, Barcelona, v. 32, n. 3, p. 493-509, 2014. Disponible en: <http://www.raco.cat/index.php/Ensenanza/ article/view/287575/375728>. Visitado el: 30 ago. 2016.

- Noticias sobre temas de astronomía en los diarios: un recurso para aprender sobre la naturaleza de la ciencia reflexivamente. Revista de Enseñanza de la Física, Córdoba, Argentina, v. 27, n. 1, p. 19-30, 2015. Disponible en: < https://revistas.unc.edu.ar/index.php/ revistaEF/article/view/11408/11884>. Visitado el: 30 ago. 2016.

GARCÍA-CARMONA, A.; ACEVEDO-DÍAZ, J. A. Learning about the nature of science using newspaper articles with scientific content: a study in initial primary teacher education. Science \& Education, Dordrecht, v. 25, n. 5-6, p. 523-546, 2016.

IZQUIERDO, M. ¿Quién tiene razón y por qué?: aprender a dudar y a decidir a partir de la lectura crítica de los textos de ciencias. (La polémica entre Pouchet y Pasteur sobre la generación de la vida). In: VIEIRA, R. M. et al. (Coord.). Ciência-tecnologia-sociedade no ensino das ciências, educação científica e desenvolvimento sustentável. Aveiro: Universidade de Aveiro, 2008. p. 129-130.

JENKINS, E. W. The history of science in British schools: retrospect and prospect. International Journal of Science Education, London, v. 12, n. 3, p. 274-281, 1990.

LATOUR, B. Pasteur y Pouchet: heterogénesis de la historia de las ciencias. In: SERRES, M. (Coord.). Historia de las ciencias. Madrid: Cátedra, 1991. p. 477-502.

MARTINS, L. A-C. P. Pasteur e a geração espontânea: uma história equivocada. Filosofia e História da Biologia, São Paulo, v. 4, p. 65-100, 2009. 
MARTINS, L. A-C. P.; MARTINS, R. A. Geração espontânea: dois pontos de vista. Perspicillum, Rio de Janeiro, v. 3, n. 1, p. 5-32, 1989. Disponible en: < http://www.ghtc.usp. br/server/pdf/lacpm-01.pdf>. Visitado el: 31 ago. 2016.

MATTHEWS, M. R. Science teaching: the contribution of history and philosophy of science. 20th anniversary revised and expanded edition. New York: Routledge, 2015.

MCCOMAS, W. F. Seeking historical examples to illustrate key aspects of the nature of science. Science \& Education, Dordrecht, v. 17, n. 2-3, p. 249-263, 2008.

Uma proposta de classificação para os tipos de aplicação da história da ciência na formação científica: implicações para a pesquisa e desenvolvimento. In: SILVA, C. C.; PRESTES, M. E. B. (Org.). Aprendendo ciência e sobre sua natureza: abordagens históricas e filosóficas. São Carlos: Tipographia Editora Expressa, 2013. p. 419-441.

MCCOMAS, W. F.; KAMPOURAKIS, K. Using the history of biology, chemistry, geology, and physics to illustrate general aspects of nature of science. Review of Science, Mathematics and ICT Education, Patras, v. 9, n. 1, p. 47-76, 2015. Disponible en: $<$ http://societyandtheory.lis.upatras.gr/index.php/review/article/download/2240/2427>. Visitado el: 30 ago. 2016.

MCMULLIN, E. Scientific controversy and its termination. In: ENGELHARDT JR., H. T.; CAPLAN, A. L. (Ed.). Scientific controversies: case studies in the resolution and closure of disputes in science and technology. New York: Cambridge University Press, 1987. p. 49-91.

MONK, M.; OSBORNE, J. Placing the history and philosophy of science on the curriculum: a model for the development of pedagogy. Science Education, Malden, v. 81, n. 4 , p. 405-424, 1997.

NEXT GENERATION SCIENCE STANDARDS. For states, by states. Washington: National Academy of Sciences, 2013. Disponible en: < http://www.nap.edu/catalog/18290/ next-generation-science-standards-for-states-by-states>. Visitado el: 31 ago. 2016.

PASTEUR, L. Estudios sobre generación espontánea. Buenos Aires: Emecé, 1944. Disponible en: < http://www.valencia.edu/ orilife/textos/Pasteur.pdf > . Visitado el: 30 ago. 2016.

RAYNAUD, D. La correspondance de F.-A. Pouchet avec les membres de l'Académie des Sciences: une réévaluation du débat sur la génération spontanée. European Journal of Sociology, Cambridge, v. 40, p. 257-276, 1999.

TOLVANEN, S. et al. How to use historical approach to teach nature of science in chemistry education? Science $\boldsymbol{\&}$ Education, Dordrecht, v. 23, n. 8, p. 1605-1636, 2014 
UNITED STATES. National Research Council. The national science education standards. Washington: National Academy Press, 1996. Disponible en: <http://www.nap. edu/catalog/4962/national-science-education-standards>. Visitado el: 31 ago. 2016.

VALLVERDÚ, J.; IZQUIERDO, M. Error y conocimiento: un modelo filosófico para la didáctica de la ciencia. Enseñanza de las Ciencias, Barcelona, v. 28, n. 1, p. 47-60, 2010. Disponible en: <http://www.raco.cat/index.php/Ensenanza/article/ view/189095/353374>. Visitado el: 31 ago. 2016.

WALLER, J. Fabulous science: fact and fiction in the history of scientific discovery. New York: Oxford University Press, 2002. 